\title{
On the Hausdorff Dimension of Topological Subspaces
}

\author{
by \\ Tomasz SZAREK and Maciej ŚLĘCZKA
}

Presented by Andrzej LASOTA

Summary. It is shown that every Polish space $X$ with $\operatorname{dim}_{\mathrm{T}} X \geq d$ admits a compact subspace $Y$ such that $\operatorname{dim}_{\mathrm{H}} Y \geq d$ where $\operatorname{dim}_{\mathrm{T}}$ and $\operatorname{dim}_{\mathrm{H}}$ denote the topological and Hausdorff dimensions, respectively.

In this note we prove that every Polish space (i.e. complete and separable metric space) $X$ has a compact subspace $Y$ such that $\operatorname{dim}_{\mathrm{H}} Y \geq \operatorname{dim}_{\mathrm{T}} X$, where $\operatorname{dim}_{\mathrm{H}}$ and $\operatorname{dim}_{\mathrm{T}}$ denote the Hausdorff dimension and the topological dimension, respectively.

From Marczewski's theorem it follows that if $X$ is a Polish space, then $\operatorname{dim}_{\mathrm{H}} X \geq \operatorname{dim}_{\mathrm{T}} X$ (see [8]). On the other hand, it is well known that for every positive integer $n$ there exists an example of a Polish space, say $E$, such that $\operatorname{dim}_{\mathrm{T}} E=n$ and $\operatorname{dim}_{\mathrm{T}} F=0$ for every compact subspace $F \subset E$ $($ see $[2,5])$.

The present paper is closely related to [7]. It fills the gap contained in the proof of Lemma 2 there. Indeed, we tacitly assumed that the measure defined in that lemma was finite. Then we used Ulam's lemma (see [1]) valid only for finite measures.

Recall now the definition of the upper Lebesgue integral. Let $A \subset \mathbb{R}$ be a Borel set. Let $f: A \rightarrow[0, \infty]$ be given. Set

$$
\mathcal{F}_{f}=\{g: A \rightarrow[0, \infty]: g \text { is Borel measurable and } g(x) \geq f(x), x \in A\}
$$

and define the upper Lebesgue integral by the formula

$$
\int_{A} f(x) d x=\inf _{g \in \mathcal{F}_{f}} \int_{A} g(x) d x .
$$

2000 Mathematics Subject Classification: Primary 54A05; Secondary 54B05.

Key words and phrases: topological dimension, Hausdorff dimension. 
The following facts can be easily derived from the above definition:

- $\bar{\int}_{A} f(x) d x=\int_{A} f(x) d x$ if $f$ is Borel measurable;

- $\bar{\int}_{A} f(x) d x \leq \bar{\int}_{A} g(x) d x$ if $0 \leq f(x) \leq g(x)$ for $x \in A$;

- $\bar{\int}_{A} f(x) d x=\int_{A} g(x) d x$ for some $g \in \mathcal{F}_{f}$;

- $\bar{\int}_{A} f(x) d x>0$ if $f(x)>0$ for every $x \in A$;

- $\bar{\int}_{A} \liminf \operatorname{in}_{n \rightarrow \infty} f_{n}(x) d x \leq \liminf _{n \rightarrow \infty} \bar{\int}_{A} f_{n}(x) d x$ for each sequence $\left(f_{n}\right)_{n \geq 1}$ of nonnegative functions.

By $B(x, r)$ (resp. $S(x, r)$ ) we denote the closed ball (resp. sphere) with centre $x$ and radius $r$.

Lemma 1. If $\operatorname{dim}_{\mathrm{T}} X \geq d+1$, where $d$ is an integer greater than or equal to -1 , then there exists $x_{0} \in X$ and $\lambda_{0}>0$ such that $\operatorname{dim}_{\mathrm{T}} S\left(x_{0}, \lambda\right) \geq d$ for every $\lambda \in\left(0, \lambda_{0}\right]$.

The proof easily follows from the definition of topological dimension (see also [6]).

Lemma 2. Suppose that $\operatorname{dim}_{\mathrm{T}} X \geq d$, where $d \in \mathbb{N} \cup\{0\}$. Then there exists a Borel probability measure $\mu$ such that

$$
\mu(B(x, r)) \leq C r^{d} \quad \text { for every } x \in X \text { and } r>0,
$$

where $C>0$ is a positive constant independent of $x$ and $r$.

Proof. We use induction on $d$. For $d=0$ condition (1) obviously holds for every Borel probability measure $\mu$. Assume that it holds for $d=m$. By Lemma 1 there exist $x_{0} \in X$ and $\lambda_{0}>0$ such that $\operatorname{dim}_{\mathrm{T}} S\left(x_{0}, \lambda\right) \geq m$ for every $\lambda \in\left(0, \lambda_{0}\right]$. Fix $\lambda \in\left(0, \lambda_{0}\right]$ and set $X_{\lambda}=S\left(x_{0}, \lambda\right)$. By the induction hypothesis there exists a Borel probability measure $\widetilde{\mu}_{\lambda}$ on $X_{\lambda}$ such that

$$
\tilde{\mu}_{\lambda}\left(B_{\lambda}(x, r)\right) \leq C_{\lambda} r^{m} \quad \text { for every } x \in X_{\lambda} \text { and } r>0,
$$

where $B_{\lambda}(x, r)$ stands for the closed ball in $X_{\lambda}$ with centre $x \in X_{\lambda}$ and radius $r$, and $C_{\lambda}$ is independent of $x$ and $r$. Without loss of generality we may assume that $C_{\lambda} \geq 1$. Define the Borel measure $\mu_{\lambda}: \mathcal{B}(X) \rightarrow[0,1]$ by the formula

$$
\mu_{\lambda}(A)=\widetilde{\mu}_{\lambda}\left(A \cap X_{\lambda}\right) /\left(2^{m} C_{\lambda}\right) \quad \text { for } A \in \mathcal{B}(X) .
$$

Clearly $\operatorname{supp} \mu_{\lambda} \subset X_{\lambda}$ and

$$
\mu_{\lambda}(B(x, r)) \leq r^{m} \quad \text { for every } x \in X \text { and } r>0 .
$$

Set

$$
\beta=\int_{\left(0, \lambda_{0}\right]}^{\bar{m}} \mu_{\lambda}(X) d \lambda
$$


and observe that $\beta>0$, by the properties of the upper Lebesgue integral. From these properties it follows that there is a decreasing sequence $\left(X_{n}\right)_{n \geq 1}$ of closed subsets of $X$ such that

$$
\int_{\left(0, \lambda_{0}\right]}^{\overline{1}} \mu_{\lambda}\left(X_{n}\right) d \lambda>\beta / 2
$$

and $X_{n}$ has a $2^{-n}$-net for each $n \in \mathbb{N}$. Indeed, let $n=1$ and let $\left\{x_{k}\right\}_{k \geq 1}$ be a dense subset of $X$. Applying the Fatou lemma for the upper Lebesgue integral to $f_{n}(\lambda)=\mu_{\lambda}\left(\bigcup_{i=1}^{n} B\left(x_{i}, 1 / 2\right)\right)$ we obtain

$$
\int_{\left(0, \lambda_{0}\right]}^{-} \mu_{\lambda}\left(\bigcup_{i=1}^{i_{1}} B\left(x_{i}, 1 / 2\right)\right) d \lambda>\beta / 2
$$

for some $i_{1} \in \mathbb{N}$. Set $X_{1}=\bigcup_{i=1}^{i_{1}} B\left(x_{i}, 1 / 2\right)$. Further, assume that we have defined $X_{1}, \ldots, X_{k}$. As before we find $i_{k+1}$ such that

$$
\int_{\left(0, \lambda_{0}\right]}^{\overline{i_{k}}} \mu_{\lambda}\left(X_{k} \cap \bigcup_{i=1}^{i_{k+1}} B\left(x_{i}, 1 / 2^{k+1}\right)\right) d \lambda>\beta / 2 .
$$

Setting $X_{k+1}=X_{k} \cap \bigcup_{i=1}^{i_{k+1}} B\left(x_{i}, 1 / 2^{k+1}\right)$ finishes the induction.

For $k \in \mathbb{N}$ and $i \in\{1, \ldots, k\}$ we define

$$
\alpha_{k, i}=\sup \left\{\mu_{\lambda}\left(X_{k}\right): \lambda \in\left((i-1) \lambda_{0} / k, i \lambda_{0} / k\right]\right\} .
$$

Let

$$
\nu_{k}=\frac{\lambda_{0}}{k} \sum_{i=1}^{k} \mu_{k, i}
$$

where $\mu_{k, i}=\mu_{\lambda_{k, i}}$ with $\lambda_{k, i} \in\left((i-1) \lambda_{0} / k, i \lambda_{0} / k\right]$ and

$$
\mu_{\lambda_{k, i}}\left(X_{k}\right) \geq \alpha_{k, i} / 2
$$

By the definition of the upper Lebesgue integral we have

$$
2 \nu_{k}\left(X_{k}\right) \geq \frac{\lambda_{0}}{k} \sum_{i=1}^{k} \alpha_{k, i} \geq \int_{0}^{\lambda_{0}} \mu_{\lambda}\left(X_{k}\right) d \lambda>\beta / 2 .
$$

Now define a positive linear functional $\Lambda: C(X) \rightarrow \mathbb{R}$ by the formula

$$
\Lambda(f)=\mathbb{L}\left(\left(\int_{X_{k}} f d \nu_{k}\right)_{k \in \mathbb{N}}\right) \quad \text { for } f \in C(X),
$$

where $\mathbb{L}$ is a Banach limit and $C(X)$ stands for the space of continuous functions $f: X \rightarrow \mathbb{R}$. From (3) it follows that $\Lambda$ is nontrivial. Let $K=$ $\bigcap_{k=1}^{\infty} X_{k}$. Observe that $K$ is a compact set and $\Lambda(f)=0$ for $0 \leq f \leq \mathbf{1}_{X \backslash K}$. 
Hence $\Lambda$ is a Riesz functional. Let $\mu_{*}$ be the Borel measure such that

$$
\Lambda(f)=\int_{X} f(x) \mu_{*}(d x) \quad \text { for } f \in C(X) .
$$

Obviously supp $\mu_{*} \subset K$. We end the proof by showing that

$$
\mu_{*}(B(x, r)) \leq 2 r^{m+1}
$$

for all $x \in X$ and $r>0$. This part of the proof is similar to the proof of Proposition 5 in [6]. It is incorporated here for convenience of the reader and to make the paper self-contained. Fix $x \in X$ and $r>0$. For $k \in \mathbb{N}$ define

$$
i(k)=\min J_{k} \quad \text { and } \quad I(k)=\max J_{k},
$$

where

$$
J_{k}=\left\{1 \leq i \leq k: B(x, r) \cap S\left(x_{0}, \lambda_{k, i}\right) \neq \emptyset\right\} .
$$

If $J_{k}=\emptyset$ we set $i(k)=I(k)=0$. Further we have

$$
\frac{\lambda_{0}}{k}(I(k)-i(k)) \leq 2 r+\frac{\lambda_{0}}{k} .
$$

On the other hand, by the construction of the measures $\nu_{k}$ we obtain

$$
\nu_{k}(B(x, r)) \leq \frac{\lambda_{0}}{k} r^{m}(I(k)-i(k)+1) \leq 2 r^{m+1}+\frac{\lambda_{0}}{k} 2 r^{m} .
$$

Fix now $\eta>0$ and let $f \in C(X)$ be such that $f(y)=1$ for $y \in B(x, r)$, $f(y)=0$ for $y \notin B(x, r+\eta)$ and $0 \leq f \leq 1$. Then

$$
\mu_{*}(B(x, r)) \leq \Lambda(f) \leq \limsup _{k \rightarrow \infty} \nu_{k}(B(x, r+\eta)) .
$$

By (4) and the fact that $\eta>0$ may be arbitrarily small, we obtain

$$
\mu_{*}(B(x, r)) \leq 2 r^{m+1}
$$

and the proof is complete.

We are in a position to formulate the main result of the paper.

Theorem 1. Suppose that $\operatorname{dim}_{\mathrm{T}} X \geq d$, where $d \in \mathbb{N} \cup\{0\}$. Then there exists a compact subspace $Y \subset X$ such that $\operatorname{dim}_{H} Y \geq d$.

Proof. From Lemma 3 it follows that there exists a Borel measure, say $\mu_{*}$, and a positive constant $C>0$ such that

$$
\mu_{*}(B(x, r)) \leq C r^{d} \quad \text { for } x \in X \text { and } r>0 .
$$

Further there exists a compact set $Y \subset X$ such that $\mu_{*}(Y)>0$, by Ulam's lemma. Then $\operatorname{dim}_{H} Y \geq d$ by Frostman's lemma (see [3, 4]).

\section{References}

[1] P. Billingsley, Convergence of Probability Measures, Wiley, New York, 1968.

[2] R. Engelking, Dimension Theory, Biblioteka Mat. 51, Warszawa, 1981 (in Polish). 
[3] K. J. Falconer, Techniques in Fractal Geometry, Wiley, Chichester 1997.

[4] O. Frostman, Potentiel d'équilibre et capacité des ensembles avec quelques applications à la théorie des fonctions, Maddel. Lunds Univ. Mat. Sem. 3 (1935), 1-118.

[5] J. van Mill, Infinite-Dimensional Topology, North-Holland, Amsterdam, 1989.

[6] J. Myjak and T. Szarek, Szpilrajn type theorem for concentration dimension, Fund. Math. 172 (2002), 19-25.

[7] J. Myjak, T. Szarek and M. Ślęczka, A Szpilrajn-Marczewski type theorem for concentration dimension on Polish space, Canad. Math. Bull. 49 (2006), 247-255.

[8] E. Szpilrajn, La dimension et la mesure, Fund. Math. 27 (1937), 81-89.

Tomasz Szarek and Maciej Ślęczka

Institute of Mathematics

Silesian University

Bankowa 14

40-007 Katowice, Poland

E-mail: szarek@intertele.pl

sleczka@ux2.math.us.edu.pl

Received July 14, 2006;

received in final form October 2, 2006 